Recent Insights into the Physics of the Sun and Heliosphere:

Highlights from SOHO and Other Space Missions

IAU Symposium, Vol. 203, 2001

P. Brekke, B. Fleck, and J. B. Gurman eds.

\title{
New Insight in Sounding the Solar Interior
}

\author{
S. Godier and J.-P. Rozelot \\ OCA-CERGA, Av. Copernic 06130 GRASSE (FRANCE)
}

\begin{abstract}
.
The solar limb is potentially a sharp spatial reference with which we can detect the effects of solar oscillations (both pression and gravitation modes), the quadrupole moment -and higher moments if any-, the true shape of the helioid (the oblateness at the first order), and changes in the solar radius. It is shown in this paper, that the accurate determination of the successive differential gravitational moments are useful to probe the solar interior. We emphasize the main reasons, mainly the accurate determination of the planetary orbits and the adjustement of the Eddington-Robertson coefficient of the PPN gravitational theory. Moreover, both the shape and the radius measurements of the Sun help to determine the solar luminosity, as empirical models of total irradiance (solely based on magnetic effects) can not explain all aspects of irradiance changes. A complete theory is still pending. The space mission Picard, currently scheduled by the end of 2004 and currently under a manufacturing process, will lead to validate our differential theory.
\end{abstract}

\section{Introduction}

This paper presents some peculiar aspects of the Sun's asphericities, mainly the gravitational moments and the oblateness. In principle, these "shape parameters" may be used to probe the solar interior. To this respect, we have developed a new modelisation, under a stratification approach, that permits to get these parameters under a differential form. The theoretical computation yields a value of the quadrupole moment of $J_{2}=1.60 .10^{-7}$ at the surface, and for the oblateness a value of $\varepsilon=8.77 .10^{-6}$. These values will be confronted in the future to space measurements, mainly from the PICARD microsatellite.

\section{Quadrupole moment and oblateness}

The solar quadrupole moment $J_{2}$ and the oblateness $\varepsilon$, two fundamental quantities, not yet known with a high degree of accuracy, are associated with the distortion of the total potential, i.e. the gravitational, rotational and magnetic (if any) potential. Their knowledge allows the determination of the shape of both the surface of the Sun and of the internal layers as soon as the distribution of mass and the velocity rates of the successive layers are known. 
The modelisation of these parameters consists in determining the solar gravitational potential using the theory of the perturbations:

$$
U_{G}=-\frac{G M_{\odot}}{R_{E}}\left[\frac{R_{E}}{r}-\sum_{n=1}^{\infty} J_{2 n}\left(\frac{R_{E}}{r}\right)^{2 n+1} P_{2 n}(\cos \theta)\right]
$$

where $G$ is the gravitational constant, $M_{\odot}$ the solar mass, $r$ the solar radial vector, $P_{2 n}$ the Legendre polynomials, $\theta$ the colatitude and $J_{2 n}$ the gravitational moments. If $y$ is the normalized expression of the perturbative gravitational potential, the expressions of both the quadrupole moment and the oblateness are the following:

$$
J_{2}=y \frac{\Omega_{\odot}^{2} r^{3}}{G M_{\odot}} \quad \text { and } \quad \varepsilon=\frac{\Omega_{\odot}^{2} r^{3}}{G M_{\odot}}\left(\frac{3}{2} y-1\right)
$$

where $\Omega_{\odot}$ is a function describing the differential rotation of the Sun. The functions given in this expression 2 are drawn on Figure 1 (Godier \& Rozelot $1999 ; 2000)$.

The two decreases observed on the profiles of the quadrupole moment $\tilde{J}_{2}$ are linked to shear layers. The first one (Fig. 1, up and left) is associated with the tachocline, where is located the passage between the radiative zone and the convective one. The second decrease (Fig. 1, up and right) is associated with the leptocline, where may also exists a passage from the convective zone to a new radiative layer located just below the surface.

The changes of curvature observed on the profiles of the oblateness are linked to solar processes. In particular, the variations visible on the second figure, seem to be the signature of the meridional flows (Gonzales-Hernandez et al. 1999) and of the zonal bands (Kosovichev 1997). Other changes of curvature can be associated with seismic events and with the store of the magnetic field.

\section{Variabilities of shape, radius and luminosity}

One of the main objectives of the next space missions devoted to the determination of the Sun's shape is to measure in real time the solar diameter in any directions together with the total irradiance (Figure 2). Such an approach permits also to study their variabilities on several timescales, and for the diameter, along the heliographic latitude. It is expected that the Picard's mission would last, at least, one solar cycle, that would lead for the first time to a comparison of the ratio irradiance/diameter over a long period of time. The determination of this ratio is of fundamental importance in Earth climatological effects induced by the Sun.

Moreover it will be possible to examine the solar surface distortions (latitudinal variability) with time and to deduce -or to refute- a temporal variation related to the solar cycle of the solar quadrupole moment and of the oblateness.

A space mission is curently scheduled to perform such measurements, under the french CNES authority: the PICARD microsatellite is expected to be launched by around 2004 (Damé et al., 2000). Another space mission SPHERIS is under consideration by an american group (NASA selection). 

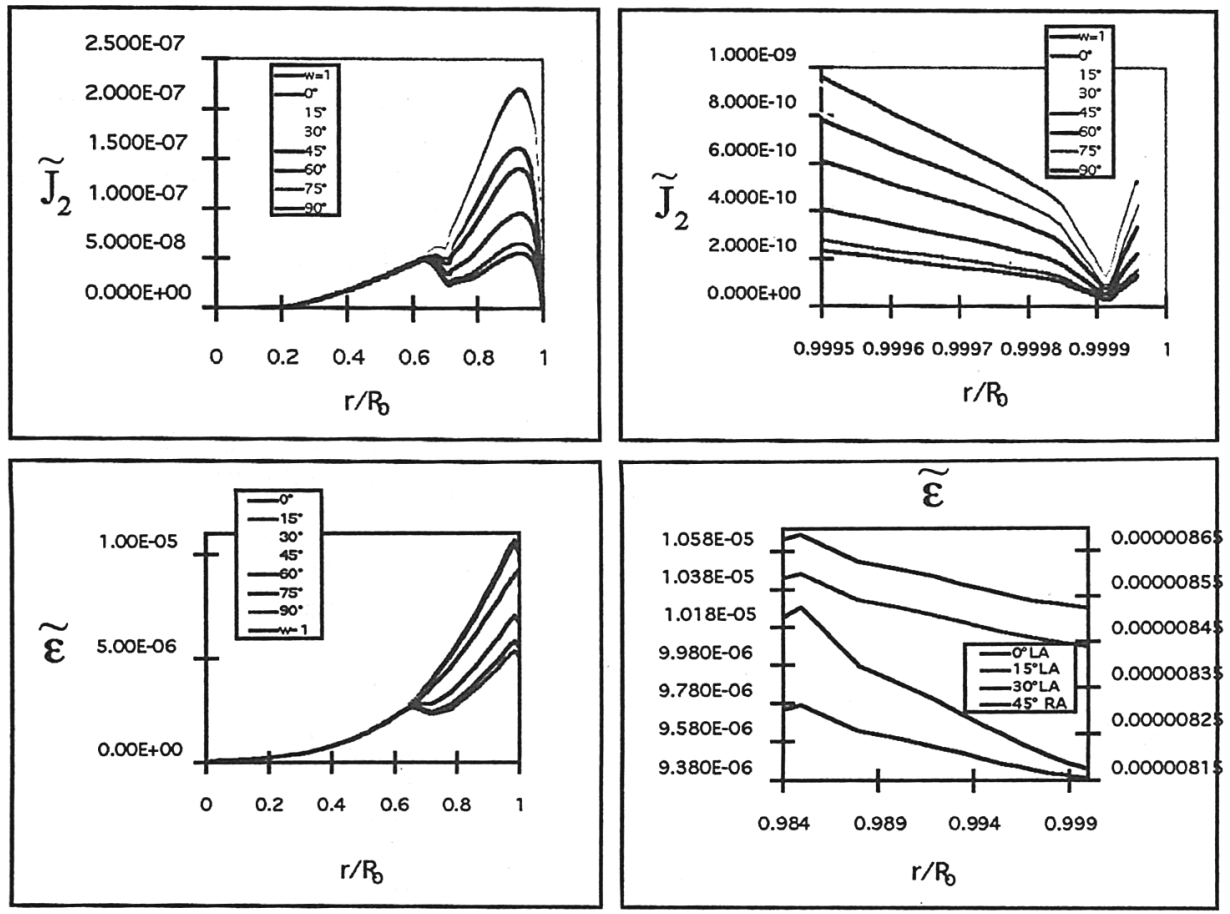

Figure 1. Quadrupole moment and oblateness of the Sun: the profiles are drawn from the core to the surface and sign the distortion of the internal layers. At the place of the tachocline and of the leptocline, two anomalies are visible on the profilesof $\tilde{J}_{2}$. Near the surface, the curvature changes in the profiles of $\varepsilon$, indicate that different physical processes occur.
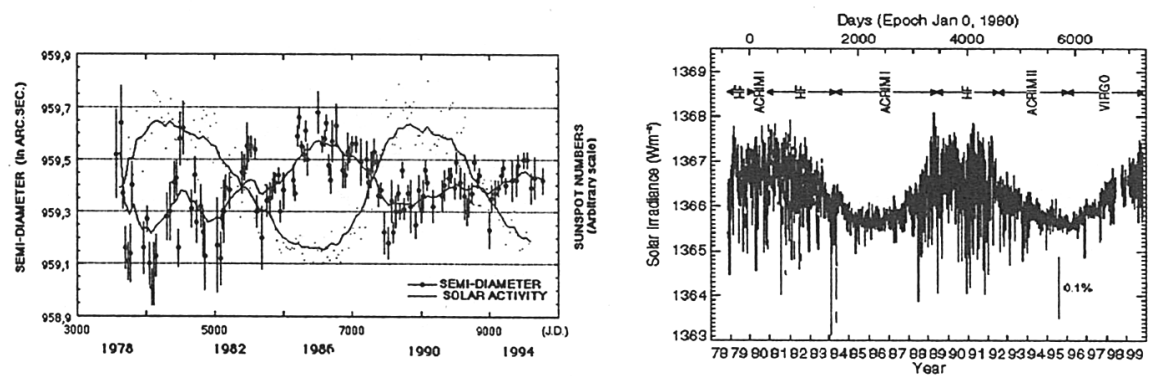

Figure 2. Temporal variation of the diameter (Laclare et al. 1996) and of the total irradiance (Fröhlich \& Lean 1998). On these figures, the diameter would be anticorrelated with the irradiance and with the solar cycle, at least untill 1994. Note that a careful inspection of the ground diameter measurements leads to a peak-to-peak variability not exceeding 100 mas, maybe less (Pap and Rozelot, 2001). 


\section{Conclusion}

Such a study of the quadrupole moment and of the oblateness is of importance, as their accurate knowledge is another way to probe the solar interior. This sounding of the solar interior allows to determinate the inhomogeneities which occur in the solar internal structure. At last, it will be possible to detect if small changes in the Sun's shape are linked with changes in the irradiance.

\section{References}

Damé, L., Cugnet, D., Joukoff, A., Rozelot, J.P. et al., 2000, Euroconference on the Solar Cycle and Terrestrial Climate, September 2000, Tenerife (S), to be published

Fröhlich, C. \& Lean, J. 1998, in IAU Symp. 185, New Eyes to See inside the Sun and Stars, ed. F. L. Deubner, J. Christensen-Dalsgaard \& D. Kurtz, 89

Godier, S. \& Rozelot, J. P. 1999, Astron. Astrophys., 350, 310

Godier, S. \& Rozelot, J. P. 2000, Astron. Astrophys., 355, 365

Gonzales-Hernandez, I. et al. 1999, ApJ, 510, L153

Kosovichev, A. G. 1999, Amer. Geophys. Union, 83

Laclare, F. et al. 1996, Solar Physics, 166, 211

Pap, J., Rozelot, J.P., Godier, S. and Varadi, F., 2001, Astron. Astrophys., submitted. 